\title{
Au/Ti-MCM-41(H)催化丙烯气相直接环氧化
}

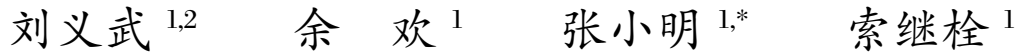 \\ ( ${ }^{1}$ 中国科学院成都有机化学研究所, 成都 610041； 2 中国科学院研究生院, 北京 100049)
}

\begin{abstract}
摘要：采用纳米组装法制备了一系列不同 $\mathrm{Ti}$ 含量的具有微孔-介孔复合结构(hybrid)的钛硅分子篮 Ti-MCM$41(\mathrm{H})$ 载体, 继而用沉积-沉淀法制得纳米金催化剂. 通过粉末 X 身线衍射 $(\mathrm{XRD})$ 、氮气等温吸附-脱附、傅里叶变 换红外(FT-IR)光谱、紫外-可见漫反射(DR UV-Vis)光谱、透射电镜(TEM)及等离子体原子发射光谱法(ICP-AES) 对催化剂进行了表征, 并考察了纳米金催化剂在氢气/氧气共存条件下丙烯气相直接氧化制环氧丙烷反应中的 催化性能. 结果表明: 合成的微孔-介孔复合结构的钛硅分子篎 Ti-MCM-41(H)具有典型的 MCM-41 结构, Ti(IV) 以高分散的形式存在于分子笁的骨架结构中. 在常压、 $423 \mathrm{~K}$ 反应温度下, 以 $\mathrm{Ti} / \mathrm{Si}$ 摩尔比为 $1 \%$ 的 Ti-MCM- 41 $(\mathrm{H})$ 为载体制备纳米金催化剂表现出了最佳的催化性能, 反应 $30 \mathrm{~min}$, 丙烯的转化率达 $5.4 \%$, 环氧丙烷的选择性 为 $74.2 \%$, 环氧丙烷的生成速率为 $73.1 \mathrm{~g} \cdot \mathrm{h}^{-1} \cdot \mathrm{kg}^{-1}$; 反应 $330 \mathrm{~min}$ 后, 丙烯的转化率为 $4.9 \%$, 环氧丙烷的选择性为 $67.3 \%$.
\end{abstract}

关键词：丙烯环氧化；微孔-介孔复合分子篮；纳米金催化剂；环氧丙烷 中图分类号：0643

\section{Direct Gas-Phase Epoxidation of Propylene over Au/Ti-MCM-41(H) Catalysts}

\author{
LIU Yi-Wu ${ }^{1,2} \quad$ YU Huan $^{1} \quad$ ZHANG Xiao-Ming ${ }^{1, *} \quad$ SUO Ji-Shuan ${ }^{1}$ \\ ('Chengdu Institute of Organic Chemistry, Chinese Academy of Sciences, Chengdu 610041, P. R. China; \\ ${ }^{2}$ Graduate University of Chinese Academy of Sciences, Beijing $\quad 100049$, P. R. China)
}

\begin{abstract}
Microporous-mesoporous hybrid Ti-MCM-41(H) was successfully synthesized by the nano-cluster assembling method. Gold nano-particles were prepared by the deposition-precipitation method. The samples were characterized by powder X-ray diffraction (XRD), nitrogen isothermal adsorption-desorption, Fourier transform infrared (FT-IR) spectroscopy, diffuse reflectance UV-Vis (DR UV-Vis) spectroscopy, transmission electron microscopy (TEM) and inductively coupled plasma emission spectrometry (ICP-AES). The catalytic performance of the gold nano-particles was evaluated by the direct gas phase epoxidation of propylene using hydrogen and oxygen. Results revealed that the Ti-MCM-41(H) had a typical mesoporous MCM-41 structure. The results also suggested that isolated Ti(IV) was introduced into the siliceous framework of the support material. Superior catalytic performance was obtained with the gold catalyst supported on Ti-MCM- $41(\mathrm{H})$ in which the Ti/Si molar ratio was $1 \%$. Propylene conversion reached $5.4 \%$ at the initial $30 \mathrm{~min}$ with $74.2 \%$ propylene oxide (PO) selectivity and produced rate $73.1 \mathrm{~g} \cdot \mathrm{h}^{-1} \cdot \mathrm{kg}^{-1}$. The conversion and PO selectivity were $4.9 \%$ and $67.3 \%$ after running $330 \mathrm{~min}$ on stream at $423 \mathrm{~K}$.
\end{abstract}

Key Words: Propylene epoxidation; Microporous-mesoporous hybrid zeolite; Nano-gold catalyst; Propylene oxide

\footnotetext{
Received: November 6, 2009; Revised: February 10, 2010; Published on Web: April 19, 2010.

"Corresponding author. Email: xm.zhang@ cioc.ac.cn; Tel: +86-28-85226215.

The project was supported by the National Natural Science Foundation of China (20703039) and Joint Scholars of Western Light Project from the Chinese Academy of Sciences.

国家自然科学基金(20703039)和中国科学院“西部之光”人才培养计划“联合学者”项目资助

(C) Editorial office of Acta Physico-Chimica Sinica
} 
环氧丙烷 $(\mathrm{PO})$ 是一种重要的有机化工原料, 是 丙烯的第三大衍生物, 主要用于生产聚醚多元醇、丙 二醇及其它化工产品 ${ }^{[1-2]}$. 目前工业上 PO 的生产方法 主要有氯醇法和间接氧化法(又称过氧化法或Halcon 共氧化法 $)^{[1-2]}$. 国内大都采用氯醇法, 但氯醇法存在 着设备腐蚀和环境污染严重等问题, 生产 $1 \mathrm{tPO}$ 约 产生 $60 \mathrm{t}$ 含氯有机废水. 共氧化法工艺过程对设备 基本无腐蚀, “三废”排放少, 但流程长, 投资大, 不宜 建中小规模的装置. 同时由于联产品多, 受市场因素 制约严重 ${ }^{[1-2]}$. 近年来, 通过科研工作者的不解努力, 在以 $\mathrm{H}_{2} \mathrm{O}_{2}$ 为氧化剂的丙烯环氧化催化剂研制方面 已取得突出进展. 特别是钛硅分子篮(如 TS-1)催化 剂在以 $\mathrm{H}_{2} \mathrm{O}_{2}$ 为氧化剂的丙烯环氧化反应中的成功 应用, 为 $\mathrm{PO}$ 的合成开辟了一条新的绿色催化途径 ${ }^{[3]}$. 其缺点为该工艺是使用稀过氧化氢溶液 $(30 \%(w))$, 成 本较高, 运输量大, 后续蒸馏负荷太大. 针对 $\mathrm{H}_{2} \mathrm{O}_{2}$ 成 本仍太高的问题, 人们随后开展了许多侧重于如何 在反应体系中原位合成 $\mathrm{H}_{2} \mathrm{O}_{2}$, 并用于丙烯环氧化反 应的研究.

以分子氧为原料的丙烯气相直接环氧化技术, 因其具有环境友好和原子利用率高等优点而日益受 到人们广泛的关注. 气-固相催化环氧化可以避免因 使用甲醇等有机溶剂所带来的环境问题, 是更为理 想的绿色化学过程. 采用沉积-沉淀法将金高度分散 于各种含 $\mathrm{Ti}$ 载体(如 $\mathrm{TiO}_{2} 、 \mathrm{TiO}_{2} / \mathrm{SiO}_{2}$ 、TS- 1 、Ti-MCM$41 / 48$ 和三维介孔 $\mathrm{Ti}-\mathrm{Si}$ 材料等)表面 ${ }^{[4-15]}$, 在 $\mathrm{H}_{2} / \mathrm{O}_{2}$ 气 氛中和较温和的反应条件下, 环氧丙烷的选择性高 达 $90 \%$ 以上. 但是, 纳米金催化剂存在一个稳定性 差的难题. 因此提高金催化剂的稳定性, 延长其使用 寿命是 $\mathrm{Au}$ 催化剂实现商业化应用需要解决的关键 问题. 为了解决纳米金催化, 以分子氧为原料的丙烯 气相直接环氧化体系中存在的丙烯转化率低 $(<5 \%)$, $\mathrm{H}_{2}$ 利用率低和催化剂失活快等问题, 人们近几年 来对该催化剂体系进行了多方位的改进. 如添加 $\mathrm{Ba}\left(\mathrm{NO}_{3}\right)_{2}$ 等碱金属或碱土金属盐助剂 ${ }^{[16-18]}$, 可以中 和催化剂表面的酸性位或提高 $\mathrm{Au}$ 的含量, 进一步 提高 PO 选择性; 采用大孔道结构的三维蠕虫状含 钛介孔分子篮作为载体 ${ }^{[16]}$, 可克服传质的限制; 通过 对催化剂表面进行硅烷化处理, 可增强催化剂表面 的疏水性, 使 PO 更容易脱附; 在反应气中添加三甲 胺以毒化载体上孤立的 Lewis 酸性位 $\mathrm{Ti}^{4+}$, 可抑制 $\mathrm{PO}$ 的后续转化, 同时也可抑制 $\mathrm{H}_{2}$ 在 $\mathrm{Au}$ 颗粒表面 上的燃烧, 提高 $\mathrm{H}_{2}$ 利用率和催化剂的稳定性 ${ }^{[[]}$. 所以
载体的性能直接决定着纳米金的催化性能和纳米 金粒子的稳定性.

为了深人理解载体对金催化剂性能的影响, 本 文采用纳米组装法(即将微孔沸石纳米簇, 又称导向 剂, 作为硅钛源组装介孔分子篮, 从而把微孔沸石的 初级和次级结构单元成功引人介孔壁中, 大幅提高 介孔分子篮的酸性和水热稳定性)合成一系列不同 $\mathrm{Ti}$ 含量的具有微孔-介孔复合结构的钛硅分子篮 $\mathrm{Ti}$ MCM-4l(H)载体,继而用沉积-沉淀法制得纳米金催 化剂, 并考察该催化剂在氢气和氧气共存条件下丙 烯气相环氧化反应中的催化性能.

\section{1 实验部分}

\section{1 载体和催化剂的制备}

以正硅酸乙酯(TEOS, 分析纯, 成都市科龙化工 试剂厂)为硅源, 钛酸四丁酯(TBOT, 分析纯, 成都市 科龙化工试剂厂)为钛源、四丙基氢氧化铵(TPAOH, 分析纯, 北京兴福精细化学研究所)和十六烷基三甲 基溴化铵 (CTAB, 分析纯, 成都市科龙化工试剂厂) 为模板剂. 首先, 制备具有微孔分子篮 TS-1 晶种的 前驱体溶液, 将含有一定量 TBOT 的异丙醇(IPA, 分析纯, 广东光华化学厂有限公司) 溶液加人 22.4 $\mathrm{mL}$ TEOS 中搅拌数分钟, 在冰水浴条件下向该混合 溶液中缓慢滴加模板剂 TPAOH 后, 再在室温下加 人适量的水并摚拌 $1-2 \mathrm{~h}$ 得澄清溶液, 将该溶液转 人高压反应釜, $100{ }^{\circ} \mathrm{C}$ 陈化 $3 \mathrm{~h}$ 得到 TS- 1 晶种的前 驱体溶液. 然后, 将模板剂 CTAB、氨水(分析纯, 广 东光华化学厂有限公司)和蒸馏水按一定比例溶解, 再将得到的 TS-1 晶种的前驱体溶液加人该溶液中, 得到物料比为 $1 \mathrm{SiO}_{2}: x \mathrm{TiO}_{2}: 0.27$ TPAOH: $0.3 \mathrm{CTAB}$ : $10 \mathrm{NH}_{3}: 200 \mathrm{H}_{2} \mathrm{O}(x=0.5 \%-5 \%)$ 的原料溶液. 室温下, 强烈搅拌反应 $24 \mathrm{~h}$, 然后将物料移至以 Telfon 衬底 的不锈钢高压釜中于 $100{ }^{\circ} \mathrm{C}$ 晶化 $24 \mathrm{~h}$, 最后冷却过 滤洗涤至 $\mathrm{pH}$ 为中性, $80{ }^{\circ} \mathrm{C}$ 干燥, 在空气中于 $540{ }^{\circ} \mathrm{C}$ 焙烧 $4 \mathrm{~h}$ 后得到具有介微孔复合结构的分子篮 $\mathrm{Ti}$ MCM-41(H). 不同 Ti 含量的样品分别标记为 Ti-41$\mathrm{H}_{x}, x$ 代表 $\mathrm{Ti} / \mathrm{Si}$ 的摩尔比 $(\%)$. 作为对比, 参照文献 [19-20]方法合成了经典微孔分子篮TS-1 和介孔分 子篮 Ti-MCM-41.

以沉积-沉淀法 ${ }^{[4]}$ 制备金催化剂. 具体制备方法 为: 取 $100 \mathrm{~g}$ 浓度为 $1.0 \mathrm{~g} \cdot \mathrm{L}^{-1}$ 的氯金酸 $\left(\mathrm{HAuCl}_{4} \cdot 4 \mathrm{H}_{2} \mathrm{O}\right.$, 分析纯, 上海久岳化工有限公司)溶液, 将其加热至 $70{ }^{\circ} \mathrm{C}$, 在加热和剧烈搅拌下滴加 $1 \mathrm{~mol} \cdot \mathrm{L}^{-1}$ 的 $\mathrm{Na}_{2} \mathrm{CO}_{3}$ 
溶液, 调节 $\mathrm{pH}$ 值为 8.8 左右; 称取 $1 \mathrm{~g}$ 所需分子篮 载体加人到该溶液中, 搅拌 $15 \mathrm{~min}$ 后, 加人助剂(5 $\mathrm{mL} 0.038 \mathrm{~mol} \cdot \mathrm{L}^{-1}$ 的 $\mathrm{Ba}^{2+}, \mathrm{Sr}^{2+}, \mathrm{Ca}^{2+}, \mathrm{Mg}^{2+}, \mathrm{K}^{+}$), 继续摚 拌 $45 \mathrm{~min}$; 停止加热, 将溶液冷却至室温后过滤, 用 $50 \mathrm{~mL}$ 去离子水洗涤. 最后在室温条件下真空干燥 $12 \mathrm{~h}$, 在空气中于 $300{ }^{\circ} \mathrm{C}$ 焙烧 $4 \mathrm{~h}$ 得到纳米金催化 剂.

\section{2 样品的表征}

XRD 分析在荷兰帕纳科公司 $\mathrm{X}^{\prime}$ pert pro MPD $\mathrm{X}$ 射线衍射仪上进行. 测定条件为 $\mathrm{Cu}$ 靶 $\left(K_{\alpha}\right)$, 管电压 $40 \mathrm{kV}$, 管电流 $40 \mathrm{~mA}$, 扫描速率为 $0.15\left(^{\circ}\right) \cdot \mathrm{min}^{-1}$, 扫 描步幅为 $0.03^{\circ} . \mathrm{N}_{2}$ 吸附-脱附分析在美国康塔公司 AUTOSORB-1 气体吸附测试仪上进行, 在液氮温 度下测定其 $\mathrm{N}_{2}$ 吸附-脱附等温曲线, 用 BET 方法计 算比表面积, 用 BJH 方法计算孔径分布及分析孔结 构. 样品测试前, 在 $300{ }^{\circ} \mathrm{C}$ 脱气 $3 \mathrm{~h}$. 在美国 Nicolet MX-1E 560 型傅里叶变换红外光谱仪上测定样品红 外光谱, $\mathrm{KBr}$ 压片. DR UV-Vis 光谱测定在北京普析 通用仪器公司 TU-1901 型紫外-可见漫反射光谱仪上 进行, 以 $\mathrm{BaSO}_{4}$ 为参比, 扫描范围 200-800 nm. 用美 国 PerKinElmer 公司 OPTIMA ICP 2000DV MKIIM6 型等离子体原子发射光谱仪分析样品组成. TEM 观 察在日立 H-900 型高分辨透射电子显微镜下进行, 加速电压 $300 \mathrm{kV}$.

\section{3 金催化剂的活性评价}

催化剂活性评价在常压固定床微型反应装置上 进行, 采用内径为 $6 \mathrm{~mm}$ 的玻璃直管反应器, 管式电 炉加热, 每次催化剂用量为 $0.30 \mathrm{~g}$, 反应气体组成用 质量流量计调控 $\mathrm{C}_{3} \mathrm{H}_{6}: \mathrm{H}_{2}: \mathrm{O}_{2}: \mathrm{N}_{2}$ 的体积比为 $1: 1: 1: 7$, 经过混合后通过催化剂床层, 空速为 $7000 \mathrm{~mL} \cdot(\mathrm{g} \cdot$

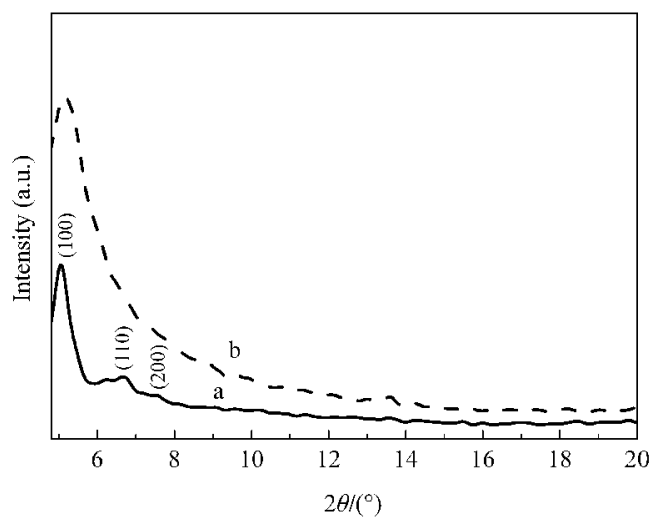

h) $)^{-1}$. 反应前对催化剂进行以下预处理: 10\%(体积 比, 下同) $\mathrm{H}_{2} / \mathrm{N}_{2}$ 和 $10 \% \mathrm{O}_{2} / \mathrm{N}_{2}$, 先后热处理 $0.5 \mathrm{~h}$, 然 后在 $\mathrm{N}_{2}$ 气氛下, 将反应温度降至 $423 \mathrm{~K}$ 后, 打开所 有反应气, 待反应进行 $0.5 \mathrm{~h}$ 后, 使用 GC7900气相 色谱(FID 检测器, TCD 检测器)对反应产物进行定 性定量分析. 采用碳平衡法计算催化剂性能, 以丙烯 转化率 $\left(X_{\mathrm{C}_{3} \mathrm{H}_{6}}\right)$ 表示催化剂活性, 用环氧丙烷的选择性 $\left(S_{\mathrm{PO}}\right)$ 表示催化剂的选择性.

\section{2 结果与讨论}

\section{1 载体和催化剂的表征}

图 1 是不同载体的 XRD 图谱. 由图中可以观 察到 TS- 1 具有典型的微孔分子篮的 MFI 结构, 采 用传统方法合成的 Ti-MCM-41 中呈现 MCM-41 的 典型特征衍射峰(100)、(110)和(200), 说明合成出的 Ti-MCM-41 为规则的六方介孔结构 ${ }^{[20]}$. 而 $\mathrm{Ti}-41-\mathrm{H}_{1}$ 以含有 TS-1 晶种的溶胶做为合成的前驱体, 这就使 得介孔的特征衍射峰有所减弱, 体现介孔长程有序 度的(110)和(200)的衍射峰强度有所减弱. 这也说明 制备的介微孔复合分子篮中, 介孔特征衍射峰强度 越低, 则微孔特征衍射峰就强, 表明介孔与微孔特征 峰强度之间存在此消彼长的关系, 即合成过程中微 孔和介孔的相对结晶度会发生变化, 微孔与介孔的 形成是相互竞争的.

由图 2 的 $\mathrm{N}_{2}$ 吸附-脱附等温线可以看出, 催化 剂吸附类型为典型的介孔分子篎的 IV 型吸附等温 线,复合分子篮(图 2(b-f))制备的催化剂在低分压段 $\left(p / p_{0}<0.1\right)$ 氮气的吸附量随着分压的升高近似线性增 加, 这是由于氮气在孔壁上发生的单分子层吸附引 起的. 随着相对压力的增大, 当 $0.2<p / p_{0}<0.4$ 时, 曲

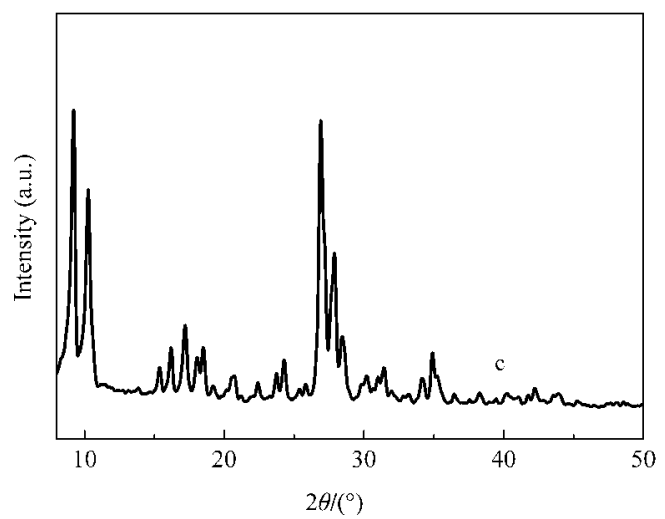

图 1 不同载体的 XRD 谱

Fig.1 XRD patterns of the different supports (a) Ti-41-H ; (b) Ti-MCM-41; (c) TS-1 


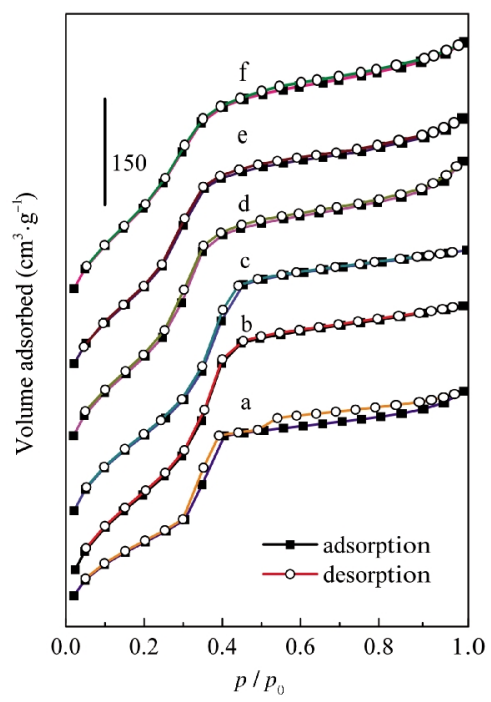

图 2 催化剂的氮气等温吸附-脱附曲线

Fig. $2 \mathrm{~N}_{2}$ adsorption-desorption isotherms of catalysts (a) Au/Ti-MCM-41; (b) Au/Ti-41- $\mathrm{H}_{0.5}$; (c) $\mathrm{Au} / \mathrm{Ti}-41-\mathrm{H}_{1}$; (d) Au/Ti-4l- $\mathrm{H}_{2}$; (e) Au/Ti-4l- $\mathrm{H}_{3}$; (f) Au/Ti-4l- $\mathrm{H}_{5}$, $x$ in $\mathrm{Au} / \mathrm{Ti}-4 \mathrm{l}-\mathrm{H}_{x}$ is molar ratio (\%) of $\mathrm{Ti} / \mathrm{Si}$.

线上尧, 吸附量有一突增, 这是由于毛细管凝聚分子 填满介孔而产生的毛细孔凝聚的结果. 曲线随后上 升缓慢, 这表明氮气在毛细管内的吸附达到饱和, 说 明复合分子篮 Ti-41-H 同时具有微孔和介孔两种孔 结构; 当 $p / p_{0}$ 接近 1 时, 曲线出现突跃, 表示在这些 压力下更大的孔被填充, 它是由颗粒间孔隙的毛细 凝聚造成的, 上尧的幅度反映出样品中大孔无定形 组分的相对含量. Ti-MCM-41 的等温线(图 2a) 在 $p /$ $p_{0}=0.2-0.4$ 之间具有明显的滞后环, 对应分子篮的 孔径在 $2.73 \mathrm{~nm}$ 左右. 从图中还可以看出, 复合分子 篮 Ti- 41-H 具有较大的比表面积, 较大的孔径和规 则的孔径分布. 结合 XRD 图可见, 该复合分子篮具 有规整单一的介孔骨架结构, 微孔结构单元已被引 进介孔分子篎的孔壁中.

表 1 列出了通过 $\mathrm{N}_{2}$ 吸附-脱附测试得到的催化 剂的孔结构参数. 复合分子篮制备的催化剂的 BET 比表面积和平均孔径均随钛含量的增加而减小, 当

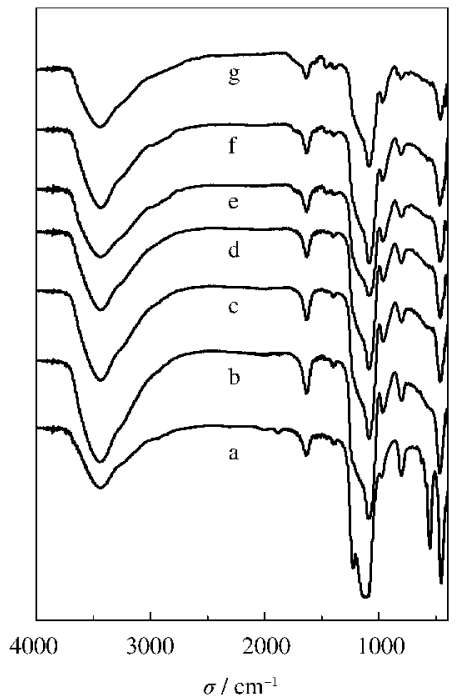

图 3 样品的傅里叶变换红外光谱

Fig.3 FT-IR spectra of the samples (a) TS-1; (b) Ti-MCM-41; (c) Ti-41-H $\mathrm{H}_{0.5}$; (d) Ti-41-H (e) Ti-4l- $\mathrm{H}_{2}$; (f) Ti-4l- $\mathrm{H}_{3}$; (g) Ti-4l- $\mathrm{H}_{5}$

钛含量较高时 $(5 \%, x)$, BET 比表面积和平均孔径略 有增加. 复合分子䇥制备的催化剂的 BET 比表面积 明显大于介孔分子篮 Ti-MCM-41 制备的催化剂的 BET 比表面积. 这可能是微孔结构单元被引进介孔 分子篮的孔壁中造成的.

为了进一步研究载体的骨架结构, 我们进行了 红外光谱测试(图 3). 从图 3 可看出, 不同钛含量的 载体 Ti-41- $\mathrm{H}_{x}$ 均在 $1090 \mathrm{~cm}^{-1}$ 附近有较强的吸收峰, 对应于硅氧四面体的不对称伸缩振动; 790 和 460 $\mathrm{cm}^{-1}$ 附近吸收峰对应 $\mathrm{Si}-\mathrm{O}-\mathrm{Si}$ 对称伸缩振动峰和 四面体 $\mathrm{Si}-\mathrm{O}$ 弯曲振动. 在 $960 \mathrm{~cm}^{-1}$ 附近均出现了 吸收峰, 说明 $\mathrm{Ti}$ 进人了合成的分子篮骨架中. 且随 着 $\mathrm{Ti}$ 含量的增加, $960 \mathrm{~cm}^{-1}$ 附近的吸收峰强度增加, 说明分子篮骨架结构的不对称性随 Ti 含量的增加 而增加. 所有复合分子篎样品(图 $3\left(\mathrm{c}-\mathrm{g}\right.$ )) 在 $570 \mathrm{~cm}^{-1}$ 左右有明显的吸收峰, 而介孔分子篮 Ti-MCM-41 (图 3b) 在 $570 \mathrm{~cm}^{-1}$ 左右没有明显的吸收峰, 微孔分 子篮 TS-1(图 3a)在 $550 \mathrm{~cm}^{-1}$ 左右有很强的吸收峰。

表 1 纳米金催化剂的表征

Table 1 Characterization of nano-gold catalysts

\begin{tabular}{|c|c|c|c|c|c|c|}
\hline Support & $x(\%)$ & $w(\mathrm{Ti})(\%)$ & $w(\mathrm{Au})(\%)$ & $S_{\mathrm{BET}} /\left(\mathrm{m}^{2} \cdot \mathrm{g}^{-1}\right)$ & $V /\left(\mathrm{cm}^{3} \cdot \mathrm{g}^{-1}\right)$ & $d / \mathrm{nm}$ \\
\hline $\mathrm{Ti}-41-\mathrm{H}_{0.5}$ & 0.5 & 0.35 & 0.07 & 1100 & 0.99 & 3.06 \\
\hline $\mathrm{Ti}-41-\mathrm{H}_{1}$ & 1 & 0.70 & 0.11 & 1062 & 0.97 & 3.04 \\
\hline $\mathrm{Ti}-41-\mathrm{H}_{2}$ & 2 & 1.31 & 0.14 & 1028 & 1.01 & 2.74 \\
\hline $\mathrm{Ti}-41-\mathrm{H}_{3}$ & 3 & 1.79 & 0.14 & 1019 & 0.92 & 2.44 \\
\hline $\mathrm{Ti}-41-\mathrm{H}_{5}$ & 5 & 3.31 & 0.19 & 1035 & 0.90 & 2.46 \\
\hline Ti-MCM-41 & 1 & 0.64 & 0.15 & 810 & 0.74 & 2.73 \\
\hline TS-1 & 1 & 0.74 & 0.05 & - & - & - \\
\hline
\end{tabular}


通常这个位置的吸收是由微孔分子篮的 $\mathrm{T}-\mathrm{O}-\mathrm{T}$ $(\mathrm{T}=\mathrm{Ti}, \mathrm{Si})$ 五元环振动引起的 ${ }^{[21]}$, 普通介孔材料在此 位置没有吸收. 因此 IR光谱结果同样说明样品含有 MFI 的微孔骨架.

图 4 给出了样品的 DR UV-Vis 光谱. 很明显, 样品在 $210 \mathrm{~nm}$ 左右有一个很尖锐的峰, 该吸收峰 是由骨架氧的成键 $2 p$ 电子轨道到骨架 $\mathrm{Ti}$ 离子的空 $d$ 轨道的电子跃迁造成的, 是分子篮骨架中四配位 钛的特征吸收峰, 这和微孔钛硅样品(TS-1)的紫外 光谱相似 ${ }^{[22]}$. 随着钛含量的升高, 此吸收峰逐渐增 强, 半峰宽变大, 表明进人骨架的钛量逐渐增多. 并 且图 4 中在 $330 \mathrm{~nm}$ 处未出现明显的电子跃迁信号, 说明各 $\mathrm{Ti}-41-\mathrm{H}_{x}$ 中不含锐钛矿 $\mathrm{TiO}_{2}$ 或非骨架钛 ${ }^{[22]}$. 与 Ti-MCM-41(图 4f)相比, Ti-41-H(图 4b)的 DR UVVis 吸收峰比较狭窄, 说明 Ti-MCM-41 (H) 中高分散 四配位 $\mathrm{Ti}(\mathrm{IV})$ 物种要更加规则, 这对于纳米金催化 剂的催化性能影响尤为重要 ${ }^{[23-24]}$.

图 5 是以复合型分子篮 $\mathrm{Ti}-41-\mathrm{H}_{1}$ 为载体制备的 金催化剂的 TEM 照片. 可以看出, 催化剂中的金粒 子在载体表面分布较为均匀且高度分散, 颗粒的大 小也比较均一, 粒子大小为 $2-4 \mathrm{~nm}$.

本文所有纳米金催化剂的金负载量及钛含量由 ICP-AES 测定, 结果列于表 1 . 从数据可知, 三种载 体(Ti-41- $\mathrm{H}_{1}$; Ti-MCM-41; TS-1)中钛的含量差别不 大, 但由于它们的结构差异, 使得金的负载量有明显 差别. 复合分子篮 Ti-41-H 做载体, 随 Ti/Si 摩尔比 的增大, 载体中钛的含量也随之增加, 金的负载量也 随载体中钛含量的增大而增大.

\section{2 催化剂的催化性能}

\subsection{1 不同载体的影响}

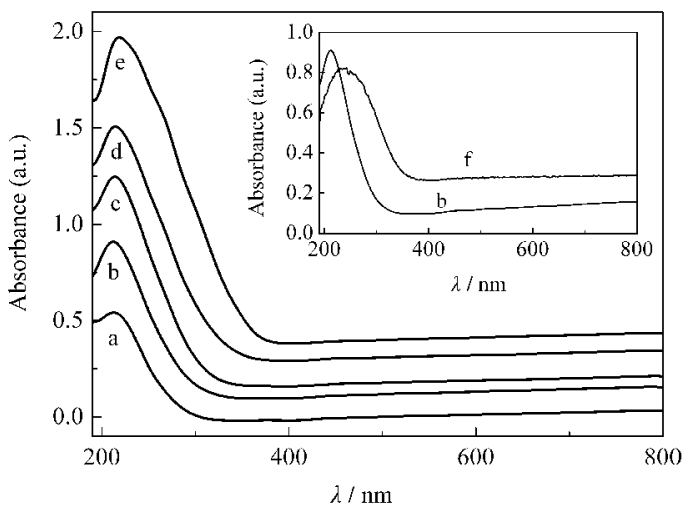

图 4 载体的紫外-可见漫反射光谱

Fig.4 DR UV-Vis spectra of supports

(a) $\mathrm{Ti}-41-\mathrm{H}_{0.5}$; (b) Ti-41- $\mathrm{H}_{1}$; (c) $\mathrm{Ti}-41-\mathrm{H}_{2}$; (d) $\mathrm{Ti}-41-\mathrm{H}_{3}$; (e) $\mathrm{Ti}-41-\mathrm{H}_{5} ;$ (f) Ti-MCM-41

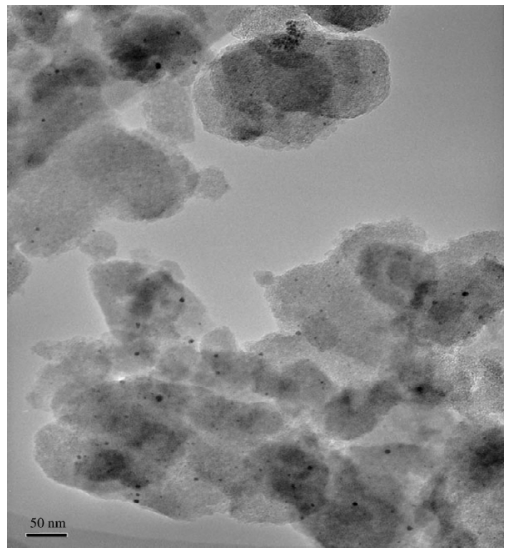

图 $5 \mathrm{Au} / \mathrm{Ti}-41-\mathrm{H}_{1}$ 催化剂的 TEM 照片

Fig.5 TEM image of the Au/Ti-41-H $\mathrm{H}_{1}$ catalyst

载体可以直接影响到活性组份的分散状况、存 在形态, 从而影响到催化剂的性能. 但到目前为止, 有关载体与催化剂性能之间的相互关系还没有一个 统一的说法. 为了进一步研究载体对 $\mathrm{Au}$ 催化剂性 能的影响，本文采用相同原料的摩尔比合成了具有 不同孔道结构的钛硅分子篮, 包括微孔分子篮TS1、介孔分子篮 Ti-MCM-41 和具有介孔-微孔复合结 构的钛硅分子篮 Ti-4l-H, 钛的含量比较接近 (表 1). 用相同条件沉积-沉淀法制备了这几种钛硅分子篮 负载的纳米金催化剂, 制备过程中都加人相同量的 $\mathrm{Ba}\left(\mathrm{NO}_{3}\right)_{2}$ 做为助剂. 但由于它们的结构差异, 使得金 的负载量有明显差别(表 1 ). 三种载体制备的金催化 剂催化性能的稳定性见图 6 . 由图可以看出, 用传统 方法合成的 Ti-MCM-41 做为载体, 丙烯的转化率

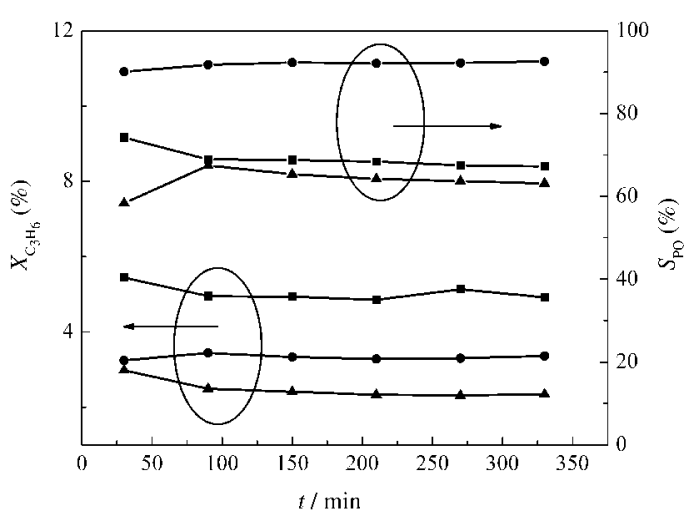

图 6 不同载体对丙烯气相环氧化性能的影响

Fig.6 Effect of different supports on the propene epoxidation in the gas phase

$\rightarrow \mathrm{Au} / \mathrm{Ti}-41-\mathrm{H}_{1} \rightarrow \mathrm{Au} / \mathrm{TS}-1 \rightarrow \mathrm{Au} / \mathrm{Ti}-\mathrm{MCM}-41$

Volume ratio of feed gas $\mathrm{O}_{2}: \mathrm{C}_{3} \mathrm{H}_{6}: \mathrm{H}_{2}: \mathrm{N}_{2}$ is 1:1:1:7, gas hourly space velocity $(\mathrm{GHSV}): 7000 \mathrm{~mL} \cdot(\mathrm{g} \cdot \mathrm{h})^{-1}$, catalyst: $0.3 \mathrm{~g}, T=423 \mathrm{~K}$; $X_{\mathrm{C}_{3} \mathrm{H}_{6}}$ : conversion of $\mathrm{C}_{3} \mathrm{H}_{6} ; S_{\mathrm{PO}}$ : selectivity of PO 
不高 $(3.0 \%)$, 且环氧丙烷的选择性也较低(58.3\%); 采用经典方法合成的 TS-1 为载体时, 丙烯的转化 率有所提高 $(3.5 \%)$, 环氧丙烷的选择性为 $92.4 \%$ 左 右, 但其生成速率约为 $58.3 \mathrm{~g} \cdot \mathrm{h}^{-1} \cdot \mathrm{kg}^{-1}$; 而以具有介 微孔复合结构的 Ti-41- $\mathrm{H}_{1}$ 为载体时, 虽然环氧丙烷 的选择性约为 $74.2 \%$, 但是丙烯的转化率有了很大 提高 $(5.4 \%)$, 环氧丙烷的生成速率也达到最高(73.1 $\left.\mathrm{g} \cdot \mathrm{h}^{-1} \cdot \mathrm{kg}^{-1}\right)$. 理论计算和实验都表明 TS- 1 的缺陷在 催化丙烯环氧化反应有重要作用 ${ }^{[14,25]}$. 本文以复合分 子篮为载体制备的纳米金催化剂的催化活性最好, 这可能是以纳米粒子为前驱体形成复合材料增加了 载体的缺陷, 介孔分子篮的孔壁中存在的微孔增加 了载体的比表面积和孔体积(表 1), 也增加了活性 $\mathrm{Ti}$ 物种, 同时降低了传质阻力. 从图 6 中还可以看出, $\mathrm{Au} / \mathrm{TS}-1$ 催化剂表现出很好的稳定性, 而 Au/TiMCM-41 和 Au/Ti-4l- $\mathrm{H}_{1}$ 随反应时间的延长, 催化活 性都有所下降, 但是以 $\mathrm{Ti}-41-\mathrm{H}_{1}$ 为载体的金催化剂 下降的速度较以 Ti-MCM-41 为载体的金催化剂要 缓慢得多, 可能是微孔结构单元被引进介孔分子篮 的孔壁中, 提高了孔壁的结晶度, 从而提高了载体的 水热稳定性. 结果表明这种介孔-微孔复合结构的钛 硅分子篮对于提高纳米金催化剂的活性和稳定性均 有利, 可见纳米组装法合成的复合分子篮载金催化 剂是较为优秀的催化剂之一.

\subsection{2 钛含量对丙烯环氧化性能的影响}

Uphade 等 ${ }^{[6,26]}$ 发现, 金催化剂中, 以介孔含钛沸 石为载体时, 其钛含量对丙烯气相环氧化性能有重 要的影响. 本文在研究 $\mathrm{Au} / \mathrm{Ti}-41-\mathrm{H}$ 催化丙烯气相直
接环氧化反应性能时, 也发现了同样的现象, 结果见 表 2. 由表可以看出, 只有适宜钛含量的 Ti-41-H 负 载 $\mathrm{Au}$ 才表现出优异的环氧化性能. 这可能因为, 当 载体钛含量高时, 载体中大量 $\mathrm{Ti}$ 物种使得锐钛矿型 二氧化钛增加, 不利于环氧化反应的进行; 而当载 体钛含量降低时, 载体中有效钛物种较少, 影响了 $\mathrm{Au}$ 的负载量, 使其没有形成足够的催化活性中心, 亦不利于环氧化反应的进行. 表 2 也列出了根据金 负载量计算的转换频率(TOF). TOF 随着钛含量的 增加而降低. 并且从表中可以看出, 钛含量摩尔比为 $1 \%$ 对应于 $\mathrm{Au}$ 的负载量为 $0.11 \%$ 时, 催化剂 $\mathrm{Au} / \mathrm{Ti}-$ 41- $\mathrm{H}_{1}$ 显示出了最佳的催化性能.

\subsubsection{Au 浓度对丙烯环氧化性能的影响}

在制备金催化剂的过程中, $\mathrm{Au}$ 溶液的浓度直接 影响着 $\mathrm{Au}$ 在载体上的负载量, 即 $\mathrm{Au}$ 浓度对金催化 剂催化丙烯气相环氧化性能有着重要的影响. 为了 考察 $\mathrm{Au}$ 负载量对催化性能的影响, 分别制备了不 同 $\mathrm{Au}$ 负载量的 $\mathrm{Au} / \mathrm{Ti}-41-\mathrm{H}$ 催化剂, 其 $\mathrm{Au}$ 的实际 负载量结果和丙烯气相环氧化性能结果见表 3 . 从 表中可以看出, 当 $\mathrm{Au}$ 浓度为 $1.0 \mathrm{~g} \cdot \mathrm{L}^{-1}$ 时, 催化剂 $\mathrm{Au} / \mathrm{Ti}-41-\mathrm{H}$ 丙烯转化率虽不是最高, 但 PO 选择性 是最好的, $\mathrm{PO}$ 的生成速率达到 $73.1 \mathrm{~g} \cdot \mathrm{h}^{-1} \cdot \mathrm{kg}^{-1}$, TOF 也最大. 综合考虑, 以 $\mathrm{Au}$ 浓度为 $1.0 \mathrm{~g} \cdot \mathrm{L}^{-1}$ 制备的 $\mathrm{Au} / \mathrm{Ti}-41-\mathrm{H}$ 丙烯环氧化性能最好.

\subsection{4 助剂的影响}

Haruta 等 ${ }^{[7,16]}$ 认为, 助剂 $\mathrm{Ba}\left(\mathrm{NO}_{3}\right)_{2}$ 能够降低催化 剂的表面酸性及促进 $\mathrm{H}_{2}$ 与 $\mathrm{O}_{2}$ 形成 $\mathrm{OOH}$, 从而提高 $\mathrm{C}_{3} \mathrm{H}_{6}$ 的转化率, 但没有给出确切的证据. 而 $\mathrm{Lu}$ 等 ${ }^{[17]}$

表 2 载体中 $\boldsymbol{n}(\mathrm{Ti}) / \boldsymbol{n}(\mathrm{Si})$ 比对丙烯气相环氧化性能的影响

Table 2 Effect of $n(\mathrm{Ti}) / n(\mathrm{Si})$ ratio on the propene epoxidation in the gas phase

\begin{tabular}{|c|c|c|c|c|c|c|}
\hline \multirow{2}{*}{ Supports } & \multirow{2}{*}{$X_{\mathrm{C}_{3} \mathrm{H}_{6}}(\%)$} & \multicolumn{3}{|c|}{$S(\%)$} & \multicolumn{2}{|c|}{ PO formation } \\
\hline & & $\mathrm{CO}_{2}$ & acetone & $\mathrm{PO}$ & $\overline{\text { rate }\left(\mathrm{g} \cdot \mathrm{h}^{-1} \cdot \mathrm{kg}^{-1}\right)}$ & TOF $\left(\mathrm{s}^{-1}\right)$ \\
\hline $\mathrm{Ti}-41-\mathrm{H}_{0.5}$ & 4.0 & 22.8 & 6.2 & 71.0 & 68.9 & 0.0928 \\
\hline $\mathrm{Ti}-41-\mathrm{H}_{1}$ & 5.4 & 20.0 & 5.8 & 74.2 & 73.1 & 0.0627 \\
\hline $\mathrm{Ti}-41-\mathrm{H}_{2}$ & 4.5 & 23.7 & 5.4 & 70.9 & 57.6 & 0.0388 \\
\hline $\mathrm{Ti}-41-\mathrm{H}_{3}$ & 3.8 & 20.7 & 4.3 & 75.0 & 51.2 & 0.0345 \\
\hline $\mathrm{Ti}-4 \mathrm{l}-\mathrm{H}_{5}$ & 4.9 & 33.0 & 4.9 & 62.1 & 54.9 & 0.0272 \\
\hline
\end{tabular}

Reaction conditions are the same as those in Fig.6. $t=30 \mathrm{~min}$; TOF: turnover frequency

表 $3 \mathrm{Au}$ 浓度对丙烯气相环氧化性能的影响

Table 3 Effect of Au concentration on the propene epoxidation in the gas phase

\begin{tabular}{|c|c|c|c|c|c|c|}
\hline \multirow{2}{*}{$\begin{array}{l}\text { Au concentration } \\
\left(\mathrm{g} \cdot \mathrm{L}^{-1}\right)\end{array}$} & \multirow{2}{*}{$X_{\mathrm{C}_{3} \mathrm{H}_{6}}(\%)$} & \multicolumn{3}{|c|}{$S(\%)$} & \multicolumn{2}{|c|}{ PO formation } \\
\hline & & $\mathrm{CO}_{2}$ & acetone & $\mathrm{PO}$ & rate $\left(\mathrm{g} \cdot \mathrm{h}^{-1} \cdot \mathrm{kg}^{-1}\right)$ & TOF $\left(\mathrm{s}^{-1}\right)$ \\
\hline 0.5 & 2.0 & 25.9 & 1.9 & 72.2 & 25.6 & 0.0345 \\
\hline 1.0 & 5.4 & 20.0 & 5.8 & 74.2 & 73.1 & 0.0627 \\
\hline 2.0 & 6.3 & 29.7 & 10.0 & 60.3 & 68.9 & 0.0260 \\
\hline
\end{tabular}

Reaction conditions are the same as those in Table 2. 
表 4 助剂对丙烯气相环氧化性能的影响

Table 4 Effect of promoters on the propene epoxidation in the gas phase

\begin{tabular}{|c|c|c|c|c|c|c|c|c|}
\hline \multirow{2}{*}{ Promoter } & \multicolumn{2}{|c|}{$w(\%)$} & \multirow{2}{*}{$X_{\mathrm{C}_{3} \mathrm{H}_{6}}(\%)$} & \multicolumn{3}{|c|}{ Selectivity (\%) } & \multicolumn{2}{|c|}{ PO formation } \\
\hline & $\mathrm{Au}$ & prometers & & $\mathrm{CO}_{2}$ & acetone & $\mathrm{PO}$ & rate $\left(\mathrm{g} \cdot \mathrm{h}^{-1} \cdot \mathrm{kg}^{-1}\right)$ & TOF $\left(\mathrm{s}^{-1}\right)$ \\
\hline- & 0.05 & - & 2.4 & 18.9 & 2.0 & 79.1 & 33.9 & 0.0639 \\
\hline $\mathrm{Ba}^{2+}$ & 0.11 & 1.97 & 5.4 & 20.0 & 5.8 & 74.2 & 73.1 & 0.0629 \\
\hline $\mathrm{Ca}^{2+}$ & 0.14 & 1.24 & 5.0 & 20.5 & 1.9 & 77.6 & 70.3 & 0.0474 \\
\hline $\mathrm{Sr}^{2+}$ & 0.16 & 0.33 & 4.9 & 21.1 & 2.7 & 76.2 & 67.9 & 0.0400 \\
\hline $\mathrm{Mg}^{2+}$ & 0.09 & 0.25 & 2.5 & 21.4 & 2.4 & 76.2 & 34.9 & 0.0367 \\
\hline $\mathrm{K}^{+}$ & 0.06 & 0.15 & 2.7 & 23.2 & 2.6 & 74.2 & 36.6 & 0.0576 \\
\hline
\end{tabular}

Reaction conditions are the same as those in Table $2 . c($ promoter $)=0.038 \mathrm{~mol} \cdot \mathrm{L}^{-1}$

通过 $\mathrm{NH}_{3}$ 的吸附分析发现, $\mathrm{Ba}\left(\mathrm{NO}_{3}\right)_{2}$ 没有降低催化 剂的酸性, 元素分析表明 $\mathrm{Ba}\left(\mathrm{NO}_{3}\right)_{2}$ 的添加能够增加 $\mathrm{Au}$ 的担载量 $50 \%$, 因而提高 $\mathrm{C}_{3} \mathrm{H}_{6}$ 的转化率. 最近他 们系统地研究了碱金属和碱土金属助剂对 $\mathrm{Au} / \mathrm{TS}-1$ 催化性能的影响 ${ }^{[18]}$, 添加 $\mathrm{K}$ 或 Cs 对金的含量影响 很小但增强了催化剂的催化性能, 碱土金属助剂增 加金的含量和增强催化剂活性, 其中 $\mathrm{Mg}$ 的效果最 佳. 金催化剂催化性能的增强主要是由于金的含量 和分散度增加. 本文主要采用了二价的碱土金属 $\left(\mathrm{Ba}^{2+}, \mathrm{Sr}^{2+}, \mathrm{Ca}^{2+}, \mathrm{Mg}^{2+}\right)$ 的硝酸盐和一价碱金属 $\left(\mathrm{K}^{+}\right)$的 硝酸盐为助剂, 且所有金属离子浓度均为 0.038 $\mathrm{mol} \cdot \mathrm{L}^{-1}$, 实验结果见表 4 . 从表中可以看出, 与没有 加助剂相比, 在催化剂中添加助剂后, PO 选择性变 化并不显著, 但丙烯的转化率则有所提高, 其中以 $\mathrm{Ba}^{2+}$ 效果最好, 丙烯的转化率 $X_{\mathrm{C}_{3} \mathrm{H}_{6}}$ 达到 $5.4 \%$, 稳定 性、TOF 值也最佳. 从表中可以看出, 随着助剂原子 半径的增大, 助剂在纳米金催化剂中的含量 $(w, \%)$ 也增大, 助剂还提高了金的利用率. 这与文献[18]报 道结果有差异. 我们认为这是由载体(微孔-介孔复 合材料, 微孔材料 $\left.{ }^{[18]}\right)$ 、中和剂 $\left(\mathrm{Na}_{2} \mathrm{CO}_{3}, \mathrm{NaOH}^{[18]}\right)$ 、沉 积-沉淀 $\mathrm{pH}$ 值 $\left(8.8,7^{[18]}\right)$ 不同导致金的含量及金的分 散度不一样而产生. 文献[18]中添加 $\mathrm{Ba}^{2+} 、 \mathrm{Sr}^{2+} 、 \mathrm{Ca}^{2+}$ 后, 金的含量太高不利于丙烯气相直接环氧化. 说明 在催化丙烯气相直接环氧化反应中, 助剂起着非常 重要的作用. 这与文献[16-17]的报道一致.

\section{3 结 论}

采用纳米组装法成功地合成了一系列不同 $\mathrm{Ti}$ 含量的具有微孔-介孔复合结构的钛硅分子篮 TiMCM-4l $(\mathrm{H})$ 载体, 它不同于 MCM-41 与 TS-1 的简 单机械混合, 而是具有微孔 TS-1 的有序孔壁和介孔 MCM-41 的六方孔道结构的复合材料. 具有微孔-介 孔复合结构的钛硅分子篮具有较大的比表面积、较
大的孔径、规则的孔径分布、较高的钛含量、高分散 的具有严格四配位结构的钛(IV)物种和较高的水热 稳定性的特点. 用沉积-沉淀法制得纳米金催化剂, 在丙烯直接气相环氧化反应中, 以 $\mathrm{Au}$ 浓度为 $1.0 \mathrm{~g}$. $\mathrm{L}^{-1}, \mathrm{Ba}\left(\mathrm{NO}_{3}\right)_{2}$ 为助剂, $\mathrm{Ti} / \mathrm{Si}$ 摩尔比为 $1 \%$ 的 Ti-MCM$41(\mathrm{H})$ 为载体, 制备纳米金催化剂, 得到最佳的催化 性能, 环氧丙烷的选择性约为 $74.2 \%$, 丙烯的转化率 达 $5.4 \%$, 环氧丙烷的生成速率为 $73.1 \mathrm{~g} \cdot \mathrm{h}^{-1} \cdot \mathrm{kg}^{-1}$. 这 些结果说明载体的水热稳定性对金催化剂的稳定性 有一定影响,纳米金催化剂 $\mathrm{Au} / \mathrm{Ti}-\mathrm{MCM}-4 \mathrm{l}(\mathrm{H})$ 是催 化丙烯在氢气、氧气共存条件下直接气相环氧化生 成环氧丙烷的优良催化剂.

\section{References}

1 Min, B. K.; Friend, C. M. Chem. Rev., 2007, 107: 2709

2 Nijhuis, T. A.; Makkee, M.; Moulijn, J. A.; Weckhuysen, B. M. Ind. Eng. Chem. Res., 2006, 45(10): 3447

3 Clerici, M .G.; Bellussi, G.; Romano, U. J. Catal., 1991, 129(1): 159

4 Hayashi, T.; Tanaka, K.; Haruta, M. J. Catal., 1998, 178(2): 566

5 Zwijnenburg, A.; Goossens, A.; Sloof, W. G.; Craje, M. W. J.; van der Kraan, A. M.; de Jongh, L. J.; Makkee, M.; Moulijn, J. A. J. Phys. Chem. B, 2002, 106: 9853

6 Uphade, B. S.; Akita, T.; Nakamura, T.; Haruta, M. J. Catal., 2002, 209(2): 331

7 Chowdhury, B.; Bravo-Suarez, J. J.; Date, M.; Tsubota, S.; Haruta, M. Angew. Chem. Int. Edit., 2006, 45(3): 412

8 Taylor, B.; Lauterbach, J.; Delgass, W. N. Appl. Catal. A-Gen., 2005, 291(1-2): 188

9 Hashmi, A. S. K.; Hutchings, G. J. Angew. Chem. Int. Edit., 2006, 45(47): 7896

10 Oyama, S. T.; Zhang, X. M.; Lu, J. Q.; Gu, Y. F.; Fujitani, T. J. Catal., 2008, 257(1): 1

11 Arcadi, A. Chem. Rev., 2008, 108(8): 3266

12 Liu, Y. W.; Yu, H.; Zhang, X. M.; Lu, J. Q.; Suo, J. S. J. Mol. Catal. (China), 2008, 22(5): 466 [刘义武, 余 欢, 张小明, 鲁继青, 索继栓. 分子催化, 2008, 22(5): 466] 
13 Yang, H. W.; Tang, D. L.; Lu, X. N.; Yuan, Y. Z. J. Phys. Chem. C, 2009, 113: 8186

14 Liu, T.; Hacarlioglu, P.; Oyama, S. T.; Luo, M. F.; Pan, X. R.; Lu, J. Q. J. Catal., 2009, 267(2): 202

15 Nijhuis, T. A.; Sacaliuc-Parvulescu, E.; Govender, N. S.; Schouten, J. C.; Weckhuysen, B. M. J. Catal., 2009, 265(2): 161

16 Sinha, A. K.; Seelan, S.; Tsubota, S.; Haruta, M. Angew. Chem. Int. Edit., 2004, 43: 1546

17 Lu, J. Q.; Zhang, X. M.; Bravo-Suarez, J. J.; Bando, K. K.; Fujitani, T.; Oyama, S. T. J. Catal., 2007, 250(2): 350

18 Lu, J. Q.; Zhang, X. M.; Bravo-Suarez, J. J.; Fujitani, T.; Oyama, S. T. Catal. Today, 2009, 147(3-4): 186

19 Thangaraj, A.; Sivasanker, S. J. Chem. Soc. Chem. Commun., 1992: 123
20 Lin, K. F.; Wang, L. F.; Meng, F. Y.; Sun, Z. H.; Yang, Q.; Cui, Y. M.; Jiang, D. Z.; Xiao, F. S. J. Catal., 2005, 235(2): 423

21 Coudurier, G.; Naccache, C.; Vedrine, J. C. J. Chem. Soc. Chem. Commun., 1982: 1413

22 Duprey, E.; Beaunier, P.; Springuel-Juet, M. A.; Bozon-Verduaz, F.; Fraissard, J.; Manoli, J. M.; Breqeault, J. M. J. Catal., 1997, 165: 22 .

23 Yap, N.; Andres, R. P.; Delgass, W. N. J. Catal., 2004, 226(1): 156

24 Cumaranatunge, L.; Delgass, W. N. J. Catal., 2005, 232(1): 38

25 Wells, D. H.; Delgass, W. N.; Thomson, K. T. J. Am. Chem. Soc., 2004, 126(9): 2956

26 Uphade, B. S.; Yamada, Y.; Akita, T.; Nakamura, T.; Haruta, M. Appl. Catal. A-Gen., 2001, 215(1-2): 137

\section{0 年全国太阳能光化学与光催化学术会议 2010 年 8 月 昆明}

中国可再生能源学会光化学专业委员会和中国化学会光化学专业委员会主办的 2010 年全国太阳能光化学与光催化学术 会议将于 2010 年 8 月 $5 、 6$ 号在云南大学召开,本次会议由云南大学承办。

本次会议是我国太阳能光化学和光催化科研工作者的一次盛会, 将全面地展示中国太阳能光化学和光催化领域所取得的 最新进展及成果, 深人地探讨太阳能光化学和光催化领域所面临的机遇、挑战及未来的发展方向, 致力于促进学术界与产业界 的沟通和联系, 促进我国太阳能光化学和光催化科学和技术的发展。大会组委会诚挚地邀请全国从事太阳能光化学和光催化研 究的同行们和本领域企业界的人士参加会议, 同时热忱欢迎从事相关研究的海外华裔同仁莅临本届盛会!

\section{一、征稿范围}

1. 光化学、纳米科学、表面、界面化学等交叉学科的研究 2 . 新型太阳能光电化学电池的研究

3. 太阳能光电化学电池的制备、组装及应用开发研究

4. 电极反应与电极反应动力学研究

5. 多相及均相光催化新反应、新构思和研究新方法

6. 光催化新材料和光催化剂制备新技术

7. 光催化反应机理及电荷转移动力学研究

8. 光催化在环境保护、新能源开发和光化学合成中的研究与成果展示

9. 自由基、中间物及瞬态过程研究新方法

10. 光催化学科发展前沿、新理论和研究热点的介绍

\section{二、征文要求}

1. 论文内容符合上述主题和国家及各单位保密规定。

2. 基本格式: A4 标准纸 1-2 页, 题目四号黑体,内容小四宋体, 打印区为高 $210 \mathrm{~mm} \times$ 宽 $150 \mathrm{~mm}$ 。论文请用激光打印,论文宣读 者姓名右上角加*号。一式两份, 寄会议联系人收, 并用 WORD 格式通过电子邮件或寄软盘将论文发送给会务组。对不符合要 求的稿件,将不予录用。

3. 被录用的论文一般不再退回修改,作者在寄论文时应做好一次性定稿的准备。论文一经录用,文责自负。论文录用与否,一概 不退,请作者自留底稿。

\section{三、重要日期}

征稿截止日期: 2010 年 5 月 31 日(以寄出邮翟为准)。录用通知(会议第二轮通知)将于 2010 年 6 月发出。

\section{四、联系方式}

联系人:李俊杰,闰智英,和 佼,何雨洋

地址: 昆明市翠湖北路 2 号云南大学化学科学与工程学院 邮编:650091

电话: 0871-5031567; 传真: 0871-5031567

QQ:911200663电邮:photochem2010@yahoo.cn; photochem2010@163.com

2010 年全国太阳能光化学与光催化学术会议组织委员会 